\title{
Compreensão de médicos residentes, cirurgiões e anestesiologistas sobre o protocolo de cirurgia segura em um hospital de ensino
}

\author{
Understanding of resident physicians, surgeons and anesthesiologists about safe surgery \\ protocol in a teaching hospital
}

Comprensión de los médicos residentes, cirujanos y anestesiólogos sobre el protocolo de cirugía segura en un hospital universitario

Taynah Ivanir da Costa de Lara ${ }^{1}$, Nelsi Salete Tonini ${ }^{1 *}$, Maristela Salete Maraschin ${ }^{1}$, Débora Cristina Ignácio da Silva ${ }^{1}$, Fabieli Borges ${ }^{1}$, Danieli Lopes ${ }^{2}$.

\section{RESUMO}

Objetivo: Identificar a percepção de médicos residentes, cirurgiões e anestesiologistas de um hospital público de ensino, acerca da Lista de Verificação de Cirurgia Segura (LVCS). Métodos: Estudo descritivoexploratório, qualitativo, com análise do Discurso do Sujeito Coletivo (DSC) em um hospital escola, com 14 sujeitos atuantes no ano de 2020 no Centro Cirúrgico, utilizando entrevista semiestruturada com cinco questões do tema. Resultados: Foram construídos 10 (dez) DSC e 13 ideias centrais, sendo: ausência de treinamento; desconhecimento do protocolo e treinamento nas residências médicas, cumprimento frágil do protocolo, diminuição dos riscos, viável/necessário, alta rotatividade de pessoal, compromisso da gestão e equipe multiprofissional, divulgação e comprometimento, orientação e cobrança, checklist digital, alteração do padrão de cores do papel impresso, realizar o checklist verbalmente e responsabilidade do profissional com o paciente. Conclusão: Necessário promover ações educativas de treinamento e aperfeiçoamento profissional, auditorias in loco, para construção dos indicadores do hospital e retorno aos profissionais.

Palavras-chave: Centro cirúrgico hospitalar, Lista de checagem, Segurança do paciente.

\begin{abstract}
Objective: Identify the perception of resident physicians, surgeons and anesthesiologists at a public teaching hospital, regarding the Safe Surgery Checklist (LVCS). Methods: Descriptive-exploratory, qualitative study, with analysis of the Collective Subject Discourse (DSC) in a teaching hospital, with 14 subjects working in the year 2020 in the Surgical Center, using semi-structured interviews with five questions on the theme. Results: 10 (ten) DSC and 13 central ideas were built, namely: lack of training; lack of protocol and training in medical residencies, weak protocol compliance, risk reduction, feasible/necessary, high staff turnover, commitment of the management and multidisciplinary team, disclosure and commitment, guidance and collection, digital checklist, color pattern change the printed paper, perform the checklist verbally and responsibility of the professional with the patient. Conclusion: It is necessary to promote educational actions for training and professional improvement, on-site audits, for the construction of hospital indicators and feedback to professionals.
\end{abstract}

Keywords: Surgery department hospital, Checklist, Patient safety.

1 Universidade Estadual do Oeste do Paraná (UNIOESTE), Cascavel - PR. *E-mail: nelsitonini@hotmail.com

${ }^{2}$ Hospital Universitário do Oeste do Paraná (HUOP), Cascavel - PR. 


\section{RESUMEN}

Objetivo: Identificar la percepción de los médicos residentes, cirujanos y anestesiólogos de un hospital universitario público sobre la Lista de Verificación de Cirugía Segura (LVCS). Métodos: Estudio descriptivoexploratorio, cualitativo con análisis del Discurso Colectivo del Sujeto (CDS) en un hospital de enseñanza, con 14 sujetos trabajando en el año 2020 en el Centro Quirúrgico, mediante entrevistas semiestructuradas con cinco preguntas sobre la temática. Resultados: Se construyeron 10 (diez) DSC y 13 ideas centrales, a saber: falta de capacitación; falta de protocolo y capacitación en residencias médicas, cumplimiento débil del protocolo, reducción de riesgos, factible / necesario, alta rotación de personal, compromiso de la gerencia y equipo multidisciplinario, divulgación y compromiso, orientación y recolección, lista de verificación digital, cambio de patrón de color, papel impreso la lista de verificación verbalmente y la responsabilidad del profesional con el paciente. Conclusión: Es necesario impulsar acciones educativas de formación y superación profesional, auditorías in loco, para la construcción de indicadores hospitalarios y retroalimentación a los profesionales.

Palabras clave: Centro quirúrgico del hospital, Lista de verificación, Seguridad del paciente.

\section{INTRODUÇÃO}

Os erros no serviço de saúde geram riscos à saúde do paciente, podendo representar desde um maior período de internamento até a morte. A cada ano, aumenta a preocupação das instituições hospitalares em elaborar medidas que diminuam a um nível mínimo a ocorrência de eventos adversos, visto que, além de gerar custos, implica na fragilidade do cuidado. No centro cirúrgico, os principais erros notificados são relacionados ao descumprimento de rotina e padrões da instituição, seguido de eventos adversos relacionados ao paciente, como queimaduras provenientes do eletrocautério e administração incorreta de medicamentos (SILVA EFM, et al., 2017).

Frente as práticas assistenciais inseguras nos serviços de saúde, é que, em 2004, a Organização Mundial da Saúde (OMS) criou a World Alliance for PatientSafety, atualmente chamado de Patient Safety Program, com o objetivo de discutir a segurança do paciente de forma a propor medidas que visem reduzir eventos adversos (OMS, 2009).

Em 2006 foi realizado o primeiro Fórum Internacional sobre Segurança do Paciente e Erro de Medicação, organizado pela Associação Mineira de Farmacêuticos em parceria com o Institute for Safe Medication Practices (ISMP) dos Estados Unidos e foi um marco para a criação do ISMP Brasil, porém, apenas em 2013 o Ministério da Saúde instituiu, por meio da Portaria MS/GM no 529, de 1으 de abril de 2013, o Programa Nacional de Segurança do Paciente (PNSP) (MINISTÉRIO DA SAÚDE, 2013).

O protocolo de cirurgia segura foi implantado no ano de 2015 no hospital estudado e, desde então, os estudos realizados se restringem a pesquisa documental acerca da adesão ao preenchimento. Sendo assim, considera-se relevante estudar o conhecimento da categoria médica acerca desta temática, com o propósito de contribuir com a instituição nos processos de avaliação, monitoramento do programa implantado e fornecimento de intervenções por parte da gestão hospitalar (MINISTÉRIO DA SAÚDE, 2014).

Nesse sentido, o objetivo do estudo foi identificar a percepção de médicos residentes, cirurgiões e anestesiologistas de um hospital público de ensino, acerca da Lista de Verificação de Cirurgia Segura (LVCS).

\section{MÉTODOS}

O cenário do estudo foi um hospital de ensino, o qual possui 238 leitos com especialidades cirúrgicas em cirurgia geral, ortopedia, ginecologia, nefrologia, cardiologia, entre outras, totalizando mais de 5 mil procedimentos cirúrgicos realizados ao ano. 
A população estudada foram 14 médicos residentes, cirurgiões e anestesiologistas atuantes no ano de 2020 que se encontravam no Centro Cirúrgico nos dias da coleta de dados. Foram inclusos na pesquisa os médicos que assinaram o Termo de Consentimento Livre e Esclarecido.

A coleta de dados foi no período de3 a 14 de agosto de 2020. Para a coleta dos dados, foi utilizado uma entrevista semiestruturada com cinco questões referentes ao tema, estas foram gravadas com o uso de aparelho celular e transcritas integralmente em documento Microsoft Word.

Para a análise dos dados, as transcrições foram interpretadas e categorizadas de acordo com o Discurso do Sujeito Coletivo (DSC), desenvolvido por Lefèvre F e Lefèvre AMC (2003). O DSC, no fim da década de 1990, possibilitou a visualização de um discurso-síntese elaborado com partes de discursos semelhantes por meio de organização e sistematização padronizada das informações colhidas durante a entrevista em seguida, o pesquisador destacou as expressões-chaves das ideias centrais e, se houver, as expressõeschave das ancoragens e as colocou nas caselas correspondentes (FIGUEIREDO MZA, et al., 2013).

Ancoragem, segundo Lefèvre F e Lefèvre AMC (2013) é uma dada teoria, ou ideologia, ou crença que o autor do discurso professa e que está sendo usada pelo enunciador para "enquadrar" uma situação específica. Após isso, as ideias centrais e ancoragens foram agrupadas com as mesmas de sentido equivalente, sendo os grupos classificados em letras: A, B, C e assim sucessivamente.

A partir desses passos, o DSC começou a ser construído. Inicialmente, as expressões-chave do mesmo grupo e coladas na coluna das expressões chaves. Com todas as expressões chaves listadas em quadros distintos representando cada grupo de ideias, no DSC, o pesquisador fará a ligação entre as expressões dos entrevistados utilizando-se de conectivos que proporcionem coesão entre as ideias (LEFÈVRE F, LEFÈVRE AMC, 2003).

A presente pesquisa foi submetida ao Comitê de Ética e Pesquisa (CEP) da Universidade Estadual do Oeste do Paraná (UNIOESTE) seguindo as normas de pesquisa com seres humanos da Resolução do Conselho Nacional de Saúde nº466/2012, sendo aprovada por meio do parecer nº 3.323.244 de 13/05/2019.

\section{RESULTADOS}

Os 14 entrevistados faziam parte das seguintes especialidades: anestesiologia (36\%), ortopedia (36\%), cirurgia geral (21\%) e neurologia (7\%), sendo que $71 \%$ eram do sexo masculino e $29 \%$ do sexo feminino. A média de idade foi 41,7 anos, $43 \%$ eram da faixa de 20 a 40 anos, $43 \%$ de 40 a 60 anos e $14 \%$ com idade superior a 60 anos. O maior tempo de atuação referido foi de 35 anos e o menor 5 meses, com média de 17,8 anos. Por fim, $86 \%$ relataram conhecer o Protocolo de Cirurgia Segura proposto pela OMS, enquanto $14 \%$ não possuíam conhecimento.

Como resultado da primeira questão: Como aconteceu o processo de implementação do protocolo de cirurgia segura nessa instituição? Você participou de algum treinamento? Construiu-se 2 discursos e 3 ideias centrais, sendo:

"Nunca participei de treinamento. Quando eu comecei já tinha uma folha para preencher, mas não era uma coisa certa. "Eles" colocaram uns quadros e tinha que preencher o quadro na sala antes de começar a cirurgia" (1ํDSC).

Ideias Centrais: Ausência de treinamento; desconhecimento do protocolo

"Lembro que foi implementado já fazem uns 5/6 anos. Participei de treinamento logo que ele foi montado. No primeiro dia da residência nós tivemos algumas palestras e foi falado sobre isso" (2 DSC).

Ideia Central: Treinamento nas residências médicas

Quanto a segunda questão: Em 2015 foi implantada, nessa instituição, o Protocolo de Cirurgia Segura. Para você, qual a viabilidade do preenchimento da lista de checagem, anexada ao prontuário do paciente. Foram construídos 3 discursos e 6 ideias centrais: 
"Existem algumas rotinas que, devido a nossa especialidade, a gente já segue mais ou menos o padrão, independente do hospital ter ou não um protocolo, as vezes eles se complementam" (1ํDSC).

Ideia Central: Cumprimento frágil do protocolo

"Eu acho que sim, totalmente viável, necessário! Igual a "Teoria do Queijo Suíço", as vezes perguntam alguma coisa lá na ala e o paciente respondeu, mas pela ansiedade do procedimento pré-operatório esqueceu de falar que toma alguma medicação ou as vezes omitiu alguma coisa pelo nervosismo e daí chega aqui, pergunta novamente. Assim conseguimos diminuir os riscos" (2ํㅗㄱ).

Ideias Centrais: Diminuição dos riscos; viável/necessário

"Eu acho que é viável, porém, várias profissionais da enfermagem saíram ou estão afastadas por atestado então as pessoas que idealizaram o programa, a grande maioria delas não está mais aqui e acho que não foi passado isso aqui porque, a instituição não abraçou o projeto" (3ํDSC).

Ideias Centrais: Rotatividade de pessoal; compromisso da gestão e equipe multiprofissional

Na terceira questão. Quais medidas você propõe para a melhoria dos índices de adesão a lista de checagem da LVCS? Surgiram 4 discursos e 5 ideias centrais:

"Primeiro a divulgação, depois o compromisso de que isto seja realizado com a equipe cirúrgica, com a equipe de enfermagem e com a equipe da anestesia"

(1ํDSC).

Ideia central: divulgação e comprometimento

"Orientação e cobrança. Acredito que as pessoas têm que ser orientadas a preencher e tem que ser cobradas" (2ํㅡㄴ.

Ideias centrais: Orientação; cobrança

"Eu acho difícil sugerir medidas, porque tudo que burocratiza, na minha opinião, só atrapalha o trabalho. Então uma maneira é fazer uma coisa mais digital ou então diminuir a burocracia" (3으).

Ideia Central: lista de checagem digital

"Eu faria em uma cor verde, um papel colorido, uma coisa que você bate o olho e já vê as alterações" (4ํDSC).

Ideia central: Alteração do padrão de cores do papel impresso

Na quarta questão: Me fale sobre a atribuição do médico no processo de preenchimento da lista de checagem em questão. Surge um único discurso, com as ideias centrais de realizar a lista de checagem verbalmente e a responsabilidade do profissional médico sobre o paciente:

"A gente sempre checa o paciente, o procedimento, de onde o paciente veio, qual vai ser o tipo de cirurgia, quem participa da cirurgia em si: o anestesista, o cirurgião, a circulante. A gente checa se ele fez antibiótico, o número de compressas, se vai ter passos críticos, se precisa de reserva ou não, se tá fazendo antibioticoterapia ou se precisa fazer. Então o médico faz essa pausa verbalmente e o técnico, vai preenchendo a lista. O médico é o responsável pelo paciente, se está correto e após a cirurgia verificar se o que aconteceu foi preenchido de forma correta" (1ํㅡㄴ DSC).

Ideias centrais: Realizar a lista de checagem verbalmente; responsabilidade com o paciente. 


\section{DISCUSSÃO}

Dos quatorze participantes da pesquisa, 12 relatam conhecer o formulário para preencher, porém, não passaram por nenhum treinamento, corroborando o estudo realizado com 202 profissionais por Silva EFM, et al. (2017), no qual a equipe médica relatou não possuir treinamento: anestesistas (62,5\%), cirurgiões $(82,4 \%)$ e residentes $(83,9 \%)$. Dessa forma, observa-se que o treinamento é uma ferramenta importante para aquisição de conhecimento para que o profissional de saúde possa promover a qualidade dos procedimentos realizados, permitindo a atuação em serviço de forma segura e efetiva.

No estudo de Santana AIS e Fonseca DGP (2017), os profissionais chamaram atenção dos gestores para a necessidade da implantação da cultura de segurança, no qual a equipe conheça, de fato, a LVCS e sua importância, bem como, tenham oportunidade de participar de treinamentos e capacitações, para assim contribuir com os índices de adesão à lista. Ademais, os profissionais trouxeram que além de cobrar a execução da lista de checagem, seja ofertado condições para que os membros passem a aderi-lo.

A cultura de segurança do paciente melhora a qualidade do cuidado e substitui a culpa e a punição por oportunidades de aprendizado com erros cometidos e cooperação da equipe, fortalecendo a ideia de responsabilidade coletiva. Além disso, a utilização do checklist é prioridade no que diz respeito à segurança do paciente e qualidade do cuidado dentro do centro cirúrgico (GUTIERRES LS, 2018).

No que se refere ao Protocolo de Cirurgia Segura, cabe à gestão hospitalar a implementação e implantação do checklist, ainda que a cultura da organização seja potencialmente dificultadora do processo. É necessário que a gestão estimule o envolvimento da equipe na adoção do hábito de preenchimento da LVCS estabelecendo união e cooperação multidisciplinar (NOVAIS RN, et al., 2016).

No segundo discurso construído, apenas dois entrevistados mencionaram a residência como palco na educação sobre o Protocolo de Cirurgia Segura. Ainda para Ribeiro L, et al. (2019), é necessário que as ações de capacitação e treinamentos sejam abordadas ainda na graduação, de forma que os estudantes tenham claro em sua formação a relevância das ações de segurança na prática cirúrgica.

Em relação ao não cumprimento do protocolo existente na instituição, em estudo realizado por Martins GS e Carvalho R (2014), a falta de colaboração da equipe médica, o timeout (processo de verificação préoperatório já com o paciente em sala operatória, é uma pausa realizada imediatamente antes do início da incisão cirúrgica), longo/repetitivo e o atraso dos médicos e das cirurgias foram fatores que dificultaram a aplicação da LVCS no Centro Cirúrgico.

Para Ribeiro L, et al. (2019), a situação de baixa adesão está relacionada com o contexto de implementação da LVCS na instituição, sendo que os resultados serão mais efetivos se a equipe médica estiver envolvida ativamente em todos os processos, assumindo funções de liderança juntamente com os demais participantes no núcleo de segurança do paciente.

Sewell M, et al. (2011), mostraram em seu estudo com 965 pacientes, que o uso da LVCS antes de um programa educacional de conscientização profissional foi de apenas 7,9\% (n480) e 47\% dos profissionais considerava que ela melhorava a comunicação da equipe. Depois do programa, a taxa de adesão subiu para $96,6 \%$ (n465) e $77 \%$ dos entrevistados referiam que o uso estava ligado à melhora da comunicação, sugerindo que ações de educação podem aumentar significativamente o uso preciso da LVCS e a percepção da equipe sobre a sua importância.

O segundo discurso dessa questão cita a Teoria do Queijo Suíço, de James Reason, professor de psicologia e especialista em fatores humanos, que propôs a imagem de um "queijo suíço" para explicar os erros que atingem o paciente durante a assistência de saúde. Para o autor, as barreiras que impedem os erros possuem fraquezas, representadas pelos buracos aleatoriamente distribuídos em cada fatia de queijo, trazendo a importância da implementação de diferentes estratégias que impeçam a ocorrência de erros e o alinhamento dos buracos do queijo suíço, permitindo que os danos atinjam o paciente (REASON J, 1990).

Quanto à rotatividade de pessoal, a maioria dos hospitais públicos brasileiros sofre com déficit no número de profissionais de enfermagem em relação ao número de leitos ofertados à população, com sobrecarga de trabalho pelas condições precárias do ambiente, com a falta de protocolos de segurança e 
com os baixos salários, levando ao aumento da probabilidade de erros e de impactos na vida do cliente e, certamente, dos profissionais (SOUZA RM, et al., 2016). Para Santana AIS e Fonseca DGP (2017), os desafios relacionados a implantação e execução do protocolo de cirurgia segura perpassam pela adesão dos profissionais ao protocolo, à comunicação na equipe e à rotatividade de profissionais, especialmente da equipe de enfermagem.

O estudo multicêntrico desenvolvido por Scherer MDA, et al. (2018), ao compararem Hospitais Universitários na Argélia, Brasil e França, evidenciaram desafios para o trabalho em saúde tais como, déficit da força de trabalho, infraestrutura e materiais, falta de leitos nos serviços de internação, excesso de atividades cronofágicas, entre outros. Para Tostes MFP e Galvão CM (2019), criar estratégias para minimizar a rotatividade de pessoal é aconselhado para um melhor desempenho da equipe.

Estudo realizado por Novais RN, et al. (2016) aponta que a prevalência de Síndrome de Burnout entre cirurgiões plantonistas em um hospital de referência para trauma foi de $46,5 \%$ (n43), além de ter sido identificado correlação entre a carga horária semanal de trabalho e a síndrome, que é caracterizada pela perda do entusiasmo pelo trabalho, sentimentos de prisão, impotência e exaustão emocional.

Nesse contexto, o terceiro DSC menciona a ausência e afastamento de profissionais incentivadores do processo como uma barreira na realização do Protocolo de Cirurgia Segura e cita a instituição como omissa no estímulo e encorajamento à adesão a lista de checagem.

Para que a ferramenta de verificação de cirurgia segura seja eficaz, é necessário que a gestão entenda e capacite os profissionais em relação a sua importância para a diminuição de riscos ao paciente, bem como incentivem a prática na sua equipe, realizem feedback e monitorem continuamente o processo para garantir um cuidado seguro. Dessa forma, o estudo de Elias ACGP, et al. (2015) mostra que, após o treinamento da equipe, houve diminuição no índice de instrumentos em branco, passando de 19 para $0 \%$ (em um total de 200 instrumentos). Em contrapartida, o preenchimento incompleto da LVCS passou de 79 para 99,5\%, atingindo a qualidade do preenchimento.

A partir da implementação do Protocolo de Cirurgia Segura, cabe à gestão realizar auditorias para monitorar a adesão dos profissionais. Para tanto, divulgar os resultados, buscar retorno da equipe como meio para elaborar estratégias em possíveis adequações do método, considerar que emergências e individualidades dos profissionais potencialmente exercem influência na efetividade da implantação, podem ser estratégias iniciais. Reconhecer esses elementos contribui para a criação de mecanismos que assegurem o sucesso e a mudança sustentável, garantindo a qualidade da assistência cirúrgica (TOSTES MFP e GALVÃO CM, 2019).

Para Conley DM, et al. (2011), a eficácia da implementação depende da forma como os líderes (anestesiologia, enfermagem e cirurgia) explicam "por que" e "como" usar a LVCS. Quando os coordenadores investem em educação extensiva sobre o assunto, reflete em altíssimos níveis de adesão. Em contrapartida, em equipes no qual não ocorre o estímulo, não há entendimento da necessidade do preenchimento, trazendo frustração, desinteresse e possivelmente abandono do método.

Uma revisão bibliográfica realizada por Tang $R$, et al. (2014), no período de 2000 a 2010, verificaram nos estudos que o processo de implementação, onde os principais fatores determinantes no sucesso da ação são: liderança eficaz, educação permanente, feedback em tempo real e contínuo, envolvimento da equipe e a LVCS como parte da cultura cirúrgica, corroborando os resultados com o presente estudo, que mostra a falta de divulgação, cobrança e feedback como dificultador do processo.

A implantação de uma lista de verificação em saúde eletrônica aumentou significativamente a transmissão fidedigna de dados, melhorou a comunicação entre a equipe, proporcionou altos níveis de satisfação nos profissionais da medicina, além de significar maior qualidade no atendimento, uma vez que possibilita a documentação completa dos dados verificados durante o seu preenchimento (DEERING SH, et al., 2015).

Além disso, mudar a cor do papel para que não se misture com o grande volume de folhas brancas do prontuário do paciente é uma boa estratégia para que a lista de checagem chame a atenção na sala 
operatória, podendo, inclusive, ser um método eficaz enquanto a instituição não conta com a LVCS no formato digital. Para este fim, sugere-se parcerias integrando hospital e universidade, por exemplo, os cursos das ciências da computação e/ou equipe de informática biomédica para o desenvolvimento de algoritmos que propiciem estudos de implementação desta tecnologia.

A realização do timeout possibilita não apenas confirmar as informações para evitar erros em paciente, ou procedimento errado, e sim identificar os quase erros (near miss), fortalecendo a barreira de segurança (MARTINS GS e CARVALHO R, 2014).

Ainda para Martins GS e Carvalho R (2014), os três fatores facilitadores da realização do timeout, de acordo com os 39 profissionais entrevistados, são: colaboração da equipe médica $(61,4 \%)$, conhecimento do protocolo $(38,5 \%)$, clareza e objetividade do protocolo $(25,6 \%)$; enquanto os três dificultadores são: falta de colaboração da equipe médica $(48,7 \%)$, protocolo longo e repetitivo $(33,3 \%)$ e atraso de médicos e cirurgias $(23,1 \%)$. Nesse sentido, comprometimento da equipe multidisciplinar na pausa cirúrgica é essencial para garantir a qualidade da assistência.

Para Ribeiro L, et al. (2019), a principal barreira encontrada no estudo foi resistência profissional na confirmação verbal nos itens de segurança de sua responsabilidade. Ressalta-se que apenas a utilização da LVCS isoladamente não é solução para promover a segurança do paciente em ambiente cirúrgico, mas sim investimento em estratégias que promovam a cultura de segurança do paciente na equipe e estreitando laços entre gestores e multiprofissionais da saúde.

Quando analisado a segurança e qualidade em cirurgia, pesquisa feita por Correia MITD, et al. (2019) mostra que dos 171 profissionais que participaram do estudo, $88,9 \%$ indicaram conhecer o projeto Cirurgia Segura do Ministério da Saúde. Quanto a falhas cirúrgicas, $81,3 \%$ indicaram já ter vivenciado falha cirúrgica grave, como corpo estranho, erro na lateralidade e falta de reserva de sangue em procedimentos em que era fundamental. Sobre os registros de tais falhas, $52 \%$ relataram não ser rotina em sua instituição de trabalho. Além disso, a pesquisa mostra que hospitais de especialidade e gerais a frequência de registros de eventos adversos é menor quando comparada a de hospitais de referência $(24,3 \%, 38,6 \%$ e $78,3 \%$, respectivamente).

Na mesma pesquisa, quando questionados sobre o uso da lista de checagem, $84,2 \%$ dos cirurgiões considerou a obrigatoriedade como uma atitude ótima e $78,4 \%$ relataram sempre apresentá-lo para sua equipe em sala. Por outro lado, apenas $3,5 \%$ dos profissionais atribui a aplicação da lista de checagem a toda equipe, enquanto a maioria $(65,5 \%)$ afirma ser atribuição do enfermeiro. Ressalta-se que 0 preenchimento do checklist é um trabalho a ser realizado em equipe, promovendo respeito e integração multiprofissional e permitindo melhores resultados na adoção e seguimento dos protocolos, corroborando estudo realizado por Tostes MFP e Galvão CM (2019) e indicando a necessidade de projetos de educação permanente reforçando o conceito de interdisciplinaridade (CORREIA MITD, et al., 2019).

$\mathrm{Na}$ Escócia, houve a implantação da ferramenta em um Hospital Universitário da cidade de Hairmyres. O grupo multiprofissional composto por médicos, enfermeiros, profissionais da radiologia, ambulatórios, paramédicos e até mesmo técnicos de informática se reúnem, diariamente, às $8 \mathrm{~h} 15 \mathrm{~min}$ para responder duas questões: "você teve algum problema de segurança do paciente hoje?" e "o que podemos fazer hoje para proteger nossos pacientes?" (HAGLEY G, et al., 2019).

\section{CONCLUSÃO}

O estudo encontrou, necessidade de treinamentos, orientações e ações educativas voltadas para a segurança do paciente. A rotatividade de pessoal, surgiu também como um dificultador para a implantação e seguimento dos protocolos. Recomenda-se, além de ações de educação permanente, inclusão da Lista de Verificação de Cirurgia Segura na coleta de dados para construção dos indicadores da instituição, os quais irão contribuir para o diagnóstico da instituição. Diante da escassez de estudos brasileiros acerca da temática, sugere-se a realização de outros estudos, para identificar o papel e o envolvimento da equipe multiprofissional no protocolo de cirurgias seguras. 


\section{REFERÊNCIAS}

1. AGARWALA AV, et. al. An electronic checklist improves transfer and retention of critical information at intraoperative handoff of care, 2015; 20(1): 96-104.

2. CONLEY DM, et al. Effective surgical safety checklist implementation, 2011; 212(5): 873-879.

3. CORREIA MITD, et al. Segurança e qualidade em cirurgia: a percepção de cirurgiões no Brasil. Revista do Colégio Brasileiro de Cirurgiões, 2019; 46(4): e2146.

4. DEERING SH, et al. Improvement in documentation using an electronic checklist for shoulder dystocia deliveries. Obstet Gynecol., 2010; 116(1): 63-66.

5. ELIAS ACGP, et al. Avaliação da adesão ao checklist de cirurgia segura em um hospital universitário público. Revista SOBECC. São Paulo, 2015; 20(3): 128-133.

6. FIGUEIREDO MZA, et al. Discurso do sujeito coletivo: uma breve introdução à ferramenta de pesquisa qualiquantitativa. Periódicos Eletrônicos em Psicologia. São Paulo, 2013; 25(1): 129-136.

7. GUTIERRES LS, et al. Boas práticas para segurança do paciente em centro cirúrgico: recomendações de enfermeiros. Revista Brasileira de Enfermagem. 2018; 71(6): 2940-2947.

8. HAGLEY G, et al. Review of alternatives to root cause analysis: developing a robust system for incident report analysis. BMJ Open Quality, 2019; 8(3): e000646.

9. LEFÈVRE F, LEFÈVRE AMC. O discurso do sujeito coletivo: 1. ed. Caxias, do Sul: Editora Educs, 2003.

10. MARTINS GS, CARVALHO R. Realização do timeout pela equipe cirúrgica: facilidades e dificuldades. Revista SOBECC. São Paulo, 2014; 19(1): 18-25.

11. MINISTÉRIO DA SAÚDE. Portaria $\mathrm{n}^{\circ}$ 529, de 1ำ de abril de 2013. Institui o Programa Nacional de Segurança do Paciente (PNSP). Brasília - DF, Brasil, 2013. Disponível em: https://bvsms.saude.gov.br/bvs/saudelegis/gm/2013/prt0529_01_04_2013.html. Acesso em: 20/11/2020.

12. MINISTÉRIO DA SAÚDE. Fundação Oswaldo Cruz, Agência Nacional de Vigilância Sanitária. Documento de referência para o Programa Nacional de Segurança do Paciente. Brasília - DF, Brasil, 2014. Disponível em: https://bvsms.saude.gov.br/bvs/publicacoes/documento_referencia_programa_nacional_seguranca.pdf. Acesso: 18/11/2020.

13. NOVAIS RN, et al. Prevalência da Síndrome de Burnout em cirurgiões plantonistas de um hospital de referência para trauma e sua correlação com carga horária semanal de trabalho: estudo transversal. Revista do Colégio Brasileiro de Cirurgiões, 2016; 43(5): 314-319.

14. ORGANIZAÇÃO MUNDIAL DA SAÚDE (OMS). Segundo desafio global para a segurança do paciente: Manual cirurgias seguras salvam vidas (orientações para cirurgia segura da OMS). Rio de Janeiro: Organização PanAmericana da Saúde, Ministério da Saúde, Agência Nacional de Vigilância Sanitária, 2009. Disponível em: https://bvsms.saude.gov.br/bvs/publicacoes/seguranca_paciente_cirurgias_seguras_guia.pdf. Acesso em: 04/11/2020.

15. REASON J. Human Error. Cambridge: Cambridge University Press, 1990; 302 p.

16. RIBEIRO L, et al. Checklist de cirurgia segura: adesão ao preenchimento, inconsistências e desafios. Revista do Colégio Brasileiro de Cirurgiões, 2019; 46(5): e20192311.

17. SANTANA AI, FONSECA DGP. As vivências na implantação do protocolo de cirurgias seguras em um hospital de pequeno porte de Sete Lagoas, Minas Gerais. Revista Brasileira de Ciências da Vida, 2017; 5(2): 1-27.

18. SCHERER MDA, et al. Desafios para o trabalho em saúde: um estudo comparado de Hospitais Universitários na Argélia, Brasil e França. Ciência e Saúde Coletiva, 2018; 23(7): 2265-2276.

19. SEWELL M, et al. Use of the WHO surgical safety checklist in trauma and orthopaedic patients. Int Orthop., 2011; 35: 897-901.

20. SOUZA RM, et al. Aplicabilidade do checklist de cirurgia segura em centros cirúrgicos hospitalares. Revista SOBECC. São Paulo, 2016; 21(4): 192-197.

21. TANG R, et al. Surgical safety checklists: a review. ANZ J Surg., 2014; 84(3): 148-154.

22. TOSTES MFP, GALVÃO CM. Processo de implementação da Lista de Verificação de Segurança Cirúrgica: revisão integrativa. Revista Latino-Americana de Enfermagem, 2019; 27(3104): 1-11. 